\title{
A self-paced CAI package for selecting statistical methods
}

\author{
PAUL E. SCHAFFNER \\ Bowdoin College, Brunswick, Maine 04011
}

\begin{abstract}
TREELAB is a package of FORTRAN IV programs, files, work sheets, and assignments for self-paced instruction in selecting statistical methods for a broad variety of empirical research purposes. It complements traditional statistics course curricula by giving students extensive practice in evaluating data sets and selecting appropriate methods according to their theoretical needs.
\end{abstract}

TREELAB is a package of FORTRAN programs, data files, problem sets, and work sheets providing practice in the selection of appropriate statistical methods for empirical research. Users begin with a description of a research situation and a goal that will require some sort of statistical analysis. Users "diagnose" the situation using a standard "diagnosis form" that seeks basic information about the statistical features of the variables involved. They then turn to a menu-driven computer progran; it poses more questions about the nature and structure of the data set and the purposes or goals for a statistical analysis. The answers to these questions determine the flow through a decision tree to one of several dozen concluding statements. Most of the statements recommend a specific statistical method; others give suggestions for reformulating theoretical questions to make them more appropriate for statistical evaluation.

TREELAB is designed for the experienced user or for the beginning student of applied statistics. It is useful as a reference tool, and especially as an instructional package. It works well in self-paced individual or small-group study settings and requires only minimal prior experience in accessing a computer through a video terminal.

One can conceive of this package as a computerized handbook for the selection of appropriate statistical methods. It serves this function, although it is not comprehensive in that regard. A second application, and the main reason for its development, is as a supplement to traditional introductory statistics course curricula. Most introductory courses in applied statistics for behavioral research emphasize hand, calculator, or computer-assisted calculation of several statistical methods, along with something of a mathematical grounding or intuitive explanation of their logics. TREELAB is designed to meet three additional objectives that emerge as students gradually orient themselves to statistical thinking and begin to assemble a repertoire of specific statistical methods. (1) It demonstrates the

This material is based upon work supported by the National Science Foundation under Grant SI:R-8001451. conceptual relations among a wide variety of methods, showing by the questions it poses both the underlying similarities that connect them and their conceptual differences. (2) It repeatedly confronts students with the germane questions involved in reformulating a theoretical question so that it can be satisfactorily addressed by a statistical analysis. Thus students are made familiar with the questions they should learn to ask themselves when facing new analytical problems and new data, and with the implications of their answers. (3) Especially when students work in groups, it allows them to discover that alternative formulations of a given problem are feasible, leading to more than one appropriate statistical analysis. Students are then in a position to become more flexible in their choices of analytic methods, as well as more clear about the relations between how a research question is phrased and which analyses can appropriately address it.

\section{ORGANIZATIONAL FORMAT FOR STATISTICAL METHODS}

In the past decade, several formal decision models have been published to aid researchers and students in choosing a statistical method (e.g., Andrews, Klem, Davidson, O'Malley, \& Rodgers, 1981; Harshbarger, 1977). Introductory statistics texts now commonly feature tree diagrams on the inside covers, or tables within, which lay out an organization of the methods they present. One explanation of why systematic approaches to statistical analysis have been late in coming may be that the vast array of methods we have available can be organized in so many different ways. For example, one can distinguish methods for experimental vs. correlational research, parametric methods vs. nonparametric methods, descriptive methods vs. inferential methods, within-subjects methods vs. betweengroups methods, methods based on the general linear model vs. all others, and so forth. Each approach requires a more or less arbitrary choice among reasonable categorization schemes; each tends to be more effective in classifying some statistical methods than others, and 
each thus tends to be incomplete. Furthermore, an organizing format covering all existing methods would be impossibly bulky and of little use because of its unwieldiness. Thus one critical task for anyone who designs such a model is to decide on boundaries for inclusion and exclusion of methods. Models implemented in ways that allow individual users to modify them so as to reflect their own ways of thinking about statistics are also preferable.

The organizing format adopted for the TREELAB project is deliberately broad in scope, including more methods than could conceivably be learned in a onesemester course. The source of the format for TREELAB is a text by Darlington (1975) that is particularly comprehensive in presenting elementary statistical methods. TREELAB articulates its organizational format for statistical methods in the main computer program users encounter. But the organizing scheme is most clearly seen in the "situation-diagnosis" form students fill out for each research situation before running the computer program (Figure 1).

The first decision TREELAB requires of its users is to categorize any statistical objective as one of four forms: sample description, population parameter estimation, hypothesis testing, or prediction. Among the idiosyncracies of this approach are the separation of correlation (sample description) from regression (prediction), and the exclusion of topics in probability theory that are clearly relevant to prediction. This four-category division, however, also has advantages. For example, it forces the user to distinguish immediately between sample and population analyses.

The second step in diagnosing a research situation according to this organization format is to determine which of four characteristics of data is relevant. These characteristics include elevation of scores, proportions or frequencies of cases, variation among scores, and covariation among variables.

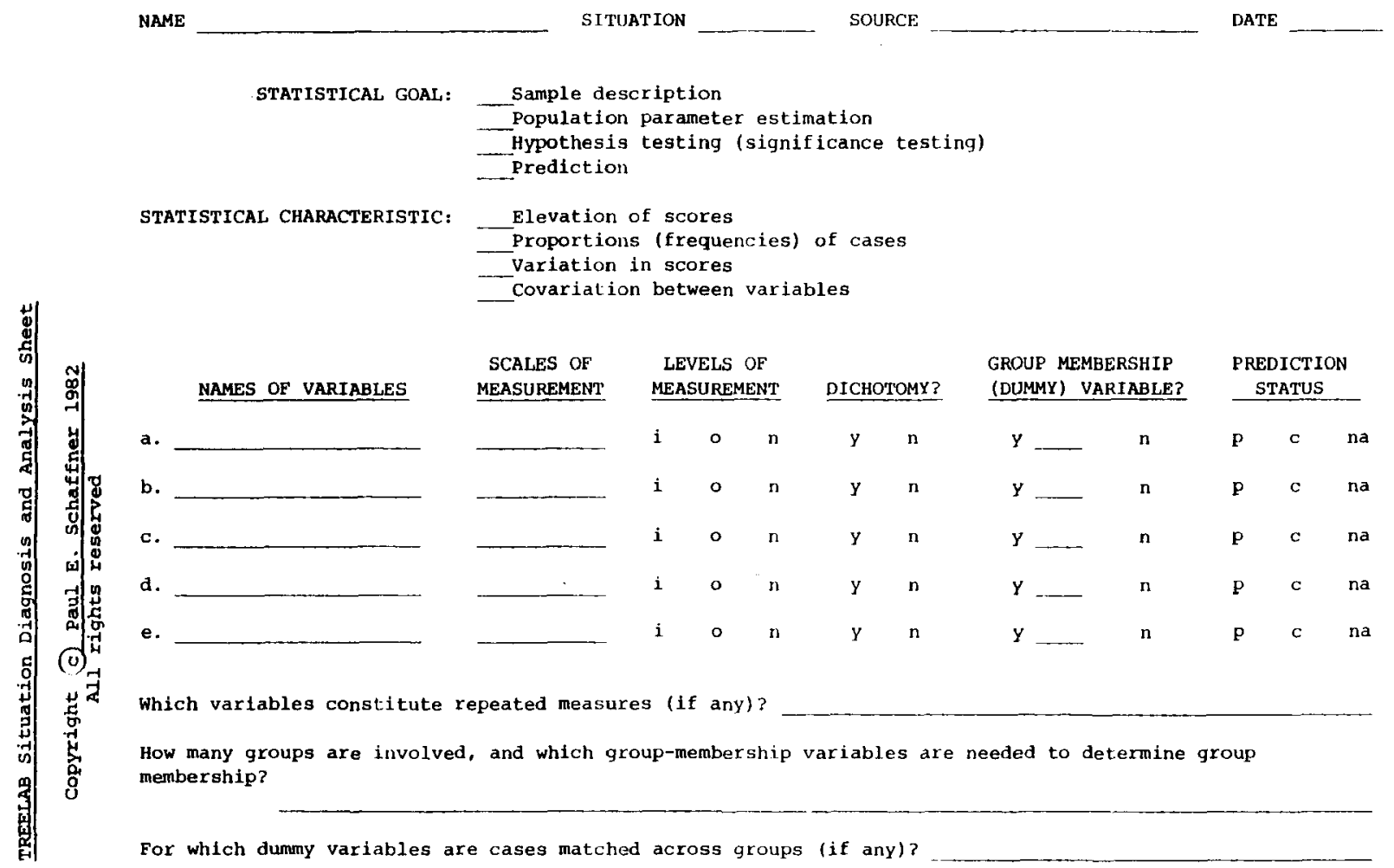

\begin{tabular}{l} 
TREE DISPLAY: \begin{tabular}{|l|l|l|l|l|l|l|l|l|l|l|l|l|l|l|l|l|} 
YOUR CHOICE: & \\
\hline
\end{tabular} \\
\hline
\end{tabular}

Final TREE recommendation:

Your comments :

Figure 1. TREELAB situation diagnosis form. 
Following the second categorization, the user determines how many variables are involved in the research situation, what the appropriate Stevens level of measurement is for each variable, how many groups are relevant, which variables indicate group membership, which are the independent and dependent variables, which variables are repeated measures, which indicate matched groups, and, in the case of statistical prediction, which variables are predictors and which are criteria.

The situation-diagnosis form seeks answers to all of these issues at the same time. The main computer program raises the first and second categorizations at the start and then selectively raises later issues as they become relevant. One important feature of TREELAB's organizational format is the emphasis placed on identifying dichotomous variables. Explicit designation of dichotomies is included because so many statistical methods require them, and because dichotomous variables usually satisfy all of the requirements of interval, ordinal, and nominal levels of measurement at once.

Once the relevant statistical goal and characteristic have been identified for a given situation, the program branches into regions that differ substantially in complexity and detail. Altogether more than 70 statistical procedures are referenced in the current implementation of the program.

\section{COMPUTER PROGRAMS AND DATA FILES}

The TREELAB package includes three data files and four executable programs, only one of which (TREE.F4) is actually used by the learner/user. The programs are written in FORTRAN IV and are now operating on a DEC 1091-KI computer at Bowdoin College. The user does not need to be aware of the data files or of the three other FORTRAN programs that organize and update the data files. Because TREE displays large quantities of text, it is recommended for use only on CRT terminals.

From the perspective of the user, the TREE program is an extended menu-driven branching decision tree. Each use of the program presents a succession of text displays on the user's screen. Branching texts end with some sort of question or choice and a brief menu of alternative answers. Terminating ("leaf") texts recommend a specific statistical method for the particular set of circumstances the user has described by his or her pattern of responses, or they indicate that no method is available or that the situation the user has described is beyond the range of coverage of the program. The user can then either exit or restart the program (the restarting option is available at all times via an asterisk response).

Each text is referenced by an index number, used by the TREE program to locate appropriate texts and by the learner/user to keep track of the successive texts confronted and the choice made for each. Most menus include a "help" response option, which, if selected, leads to a help text and then back to the previous text and menu. A few special answer-format branching texts call for detailed information (such as about sample sizes) incompatible with a standard menu answer format. In these cases, a subroutine preprocesses whatever information is requested, converting it into a menu pseudochoice and returning that "choice" to the main driver program.

Currently, 237 text displays are available in the package. These include 88 normal branching texts, 4 special answer-format branching texts, 112 terminating texts, and 33 help texts. All are stored in file TEXTS.DAT and are randomly accessible by the main driver program (TREE.F4). Texts are stored in numerical order, but their index numbers are arbitrarily assigned and do not carry information about the logical structure of the decision tree. Texts can be any number of lines long; the current set averages just under 6 lines/text, including response menus. The text data file is line blocked with 72 characters plus index numbers in Columns 1.3 of each line and an asterisk (required by the DEC-10 FORTRAN random file-access utility) in Column 72.

The statistical logic of the TREELAB package is contained in its branching instructions. These are stored in file BRANCH.DAT, containing one line of coded branching instructions for each logical decision point or "node" in the tree. Line numbers of the file identify each node, but there is no logical connection between the node numbers and the index numbers of texts stored in TEXTS.DAT.

At any given time, the TREE program is "located" on one of the branching instruction (node) lines of BRANCH.DAT. The first three characters in that line give the index number of the text currently on display at the user's terminal. The fourth character designates the type of text (normal branching, special answer-format branching, terminating, or help). Then, 10 successive numbers designate other nodes as destinations, to 1 of which TREE will move when the user enters a number (0 through 9) from the menu on display at the terminal. The final entry in each node line in BRANCH.DAT is its line or node number. While the user answers consecutive questions about the research problem, TREE moves from node to node in BRANCH.DAT, each new node line indicating which text to display and which succeeding node to proceed to for any menu option the user might choose.

Separating the logical structure of the decision tree from the list of texts allows the programmer to use any given text repeatedly, each time called by a different node of the tree. This practice saves considerable storage space in the display text file by avoiding unnecessary duplication of text material. In TREELAB's current 
configuration the 237 texts are referenced a total of 327 times by nodes in BRANCH.DAT. Of the 327 nodes, 141 display normal branching texts, 4 display special answer-format branching texts, 112 display terminating texts, and 70 display help texts. Branching texts are referenced up to eight times by different nodes, and help texts, up to 10 times.

The appearance of the same texts at several points in the logical structure of TREELAB is consistent with a major underlying assumption of the package: that often, the same questions must be answered to reformulate apparently disparate statistical problems so that an appropriate analytic method can be chosen. Thus it frequently happens that two people running TREE will arrive at the same current display and choice from different prior displays and choices; their current choices, even if numerically identical, will then lead to different subsequent texts. In repeated use of the package, the learner/user comes to recognize the recurrence of common issues in different research contexts. Such recognition is of course one of the primary educational objectives of the package.

The third data file of the package (START.DAT) is a list of the starting line numbers in TEXTS.DAT of each successive text. TREE checks this list prior to each random access of the TEXT.DAT file to read the current text display. One of the supporting programs, START.F4, updates START.DAT whenever modifications are made to any texts. Another supporting program, 76CHAR.F4, extends all edited or new texts to 76 characters/line and adds an asterisk prior to line blocking. The third supporting program, MLTCHG.F4, maintains and updates the branching structure of the package as stored in BRANCH.DAT. In its current configuration, the three data files of TREELAB require $21 \mathrm{~KB}$ (TEXTS.DAT), $4 \mathrm{~KB}$ (BRANCH.DAT), and .4 KB (START.DAT) of storage. The main driver program (TREE.F4) requires $2 \mathrm{~KB}$ (12.5 KB in executable form), including 140 lines of code and 190 lines of documentation. The supporting programs together require about $1.6 \mathrm{~KB}$ of storage. A help file (TREE.HLP) contains another 285 lines of documentation and instructions.

\section{USE OF THE TREELAB PACKAGE}

TREELAB is now in its 3rd year of development and use at Bowdoin. Students in the introductory statistics course began using the package after 7 weeks of classroom and laboratory work covering fundamental concepts of data and descriptive statistics, plus computer access skills. Each week, students receive a set of six hypothetical research "situations." Each situation describes a data set and a theoretical question of interest to the researcher, with varying degrees of clarity and completeness. The situations presented each week are deliberately chosen to reflect a broad array of statistical applications, in many cases leading students to choose methods not yet covered in lecture or reading assignments. For example, students encounter situations requiring significance tests before those significance tests are covered in class. Because students have already studied the statistical concepts pursued on the TREE$\mathrm{LAB}$ situation diagnosis form, this approach allows them to discover for themselves methods the procedures for which will be presented later in class. In addition, the TREELAB repertoire of methods is much broader than the curriculum of a one-semester course can cover, so the laboratory exercises can also be used to make students aware of the existence of methods that they will come to study only in more advanced courses. Occasionally, late in the semester, students are also given reciprocal problems consisting of a recommended statistical method and various characteristics of a data set. They are then asked to imagine a hypothetical "situation" that might yield those circumstances.

Students work in small groups to complete their TREELAB assignments. They individually diagnose each situation and then meet together to compare diagnoses, discuss and reconcile differing interpretations, and run the TREE program jointly. They record successive text index numbers and menu choices on their diagnosis forms. They note TREE's terminating recommendation and record any comments they have about their handling of that situation. Often, student teams will evaluate a given situation in more than one way and then run the TREE program twice or more for a given situation, to discover the statistical consequences of alternative formulations of a research question.

Using the computer in conjunction with the situationdiagnosis form and having students work in small groups together yield at least four pedagogic advantages to this approach. First, the program is always ready to head off in any statistical direction. With a proper mix of situation assignments, students cannot defeat the purpose of the package by inferring most of their choices from the topic of whatever text chapter or lecture is current. Second, displaying the texts and menus on a CRT rather than in print on paper avoids the intimidating impact of the complete flowchart, which covers three office walls on Kraft paper. It also stymies any tendency to look ahead rather than thoughtfully answer the question at hand. With TREE controlling presentations, the next display is not available until the current choice has been made. Third, having students work in small groups appears to make the assignments more interesting, and it increases the laboratory's educational value by forcing students to confront each other's differing interpretations of a situation when differences arise. Fourth, the completed diagnosis sheets contain detailed records of students' interpretations of each situation, as well as their sequential menu selections. These are useful to an instructor in identifying areas of general 
confusion or ambiguous texts, and in following the reasoning of any student who may have difficulty with an assignment.

By the end of the semester and the series of TREE$\mathrm{LAB}$ assignments, students are not expected to have memorized the logical structure of the package. Through repeated encounters, however, they are expected to have learned to pose for themselves the fundamental questions of data organization and research goals that need answers before appropriate methods can be chosen. TREELAB also acquaints students with the existence of possibilities other than the ones most often used (includ- ing advanced multivariate statistics), thus expanding their repertoire of ways of approaching data analysis.

\section{REFERENCES}

Andrews, F. M., Klem, L., Davidson, T. N., O'Malley, P. M., \& RODGERs, W. L. A guide for selecting statistical techniques for analyzing social science data (2nd ed.). Ann Arbor: University of Michigan, 1975.

Darlinaton, R. B. Radicals and squares (2nd ed.). Ithaca, N.Y: Logan Hill, 1975.

Harshbaroen, T. R. Introductory statistics: A decision map (2nd ed.). New York: Macmillan, 1977. 